\section{Perda auditiva induzida pelo ruído em trabalhadores de metalúrgica}

\author{
Simone Adad Araújo ${ }^{1}$
}

Resumo
I

ntrodução: A perda auditiva induzida pelo ruído é uma patologia insidiosa cumulativa, que cresce ao longo dos anos de exposição ao ruído associado ao ambiente de trabalho. Objetivos: Identificar e quantificar a ocorrência de alterações auditivas sugestivas de Perda Auditiva Induzida pelo Ruído e os principais sintomas otorrinolaringológicos referidos pelos trabalhadores. Forma de estudo: prospectivo clínico randomizado. Casuística e métodos: Pesquisa realizada no período de janeiro a março de 2000 com 187 trabalhadores de indústria metalúrgica no município de Goiânia, avaliados por médicos otorrinolaringologistas através de roteiro de entrevista e audiometria ocupacional. Resultados: Foram obtidas audiometrias ocupacionais sendo: $21 \%$ sugestivas de PAIR, $72 \%$, normais e $7 \%$, sugestivas de outras doenças auditivas. Os sintomas auditivos mais freqüentes foram: dificuldade de compreensão da fala, 12\%; hipoacusia, 7\%; tinitus, 13\%; sensação de plenitude auricular, 4\%; otorreia, 6\%; tonturas, 12\%. Conclusão: Concluímos que em metalúrgica há ocorrência de alterações auditivas sugestivas de Perda Auditiva Induzida pelo Ruído e queixa de sintomas otorrinolaringológicos significativos.

Noise induced heariing loss in metallurgical workers

Palavras-chave: perda auditiva, ruído ocupacional, metalúrgicos.

Key words: hearing loss, occupational noise, metallurgist. ntroduction: Noise induced hearing loss is a cumulative pathological disease that increases over the years with exposure to noise associated with the work atmosphere. Objectives: To identify and quantify the occurrence of hearing loss suggestive of Noise Induced Hearing Loss (NIHL) and the principle otolaryngological symptoms referred to by workers. Study design: prospective clinical randomized. Material and method: Research was carried out from January to March of 2000 with 187 metallurgical workers in the city of Goiânia, who were evaluated by otolaryngologists by means of a combination of interviews and occupational hearing exams. Results: Occupational hearing tests results were: $21 \%$ suggestive of NIHL, $72 \%$ normal, and $7 \%$ suggestive of other hearing impairments. The most frequent hearing symptoms were: difficulty of comprehension in speaking $12 \%$, Hearing loss $7 \%$, Tinnitus $13 \%$, Auricular fullness feeling 4\%, Effusion 6\%, and Dizziness $12 \%$. Conclusion: We concluded that in metallurgist exists an occurrence of hearing loss suggestive of NIHL and complaints of significant otolaryngological symptoms.

${ }^{1}$ Especialista em Medicina do Trabalho pela Faculdade de Medicina de Itajubá. Mestre em Otorrinolaringologia pela Faculdade de Medicina de Ribeirão Preto da Universidade de São Paulo e Doutoranda em Otorrinolaringologia pela Faculdade de Medicina da Universidade de São Paulo. 


\section{INTRODUÇÃO}

O ruído é um tipo de som que provoca efeitos nocivos no ser humano, sendo uma sensação auditiva desagradável que interfere na percepção do som desejado. A perda induzida pelo ruído é uma patologia cumulativa e insidiosa, que cresce ao longo dos anos de exposição ao ruído associado ao ambiente de trabalho. É causada por qualquer exposição que exerça uma média de $90 \mathrm{~dB}$, oito horas por dia, regularmente por um período de vários anos. A perda auditiva induzida pelo ruído é uma doença de caráter irreversível e de evolução progressiva passível totalmente de prevenção.

Relatos de que operários que trabalham em ambiente ruidoso eram sujeitos a hipoacusia e surdez ocupacional foram feitos por Mocelin ${ }^{7}, 1951$, que já sugeria a profilaxia individual no ambiente de trabalho e uso de proteção auditiva durante o trabalho.

Os quatro possíveis mecanismos de lesões do órgão de Corti foram relatados por Lim e Dunn', 1979, ocorrem preferentemente na espira basal da cóclea, na área responsável pelo som de 3 a $6 \mathrm{kHz}$, independente do espectro de freqüência do ruído agressor sendo:

1) insuficiência vascular na região coclear responsável pela audição a 4 kHz;

2) a velocidade de propagação da onda sonora é muito alta e faz com que a amplitude de deslocamento no ducto coclear comece a crescer na região de $4 \mathrm{kHz}$;

3) a estrutura anatômica da cóclea causa colisão do fluido na primeira curva coclear;

4) características de ressonância do canal auditivo provocariam a lesão na região citada?

Relatado por Seligman e Ibañez ${ }^{12}$, 1993, a exposição crônica ao ruído produz deterioração auditiva lenta, progressiva e irreversível com características de disacusia neurossensorial geralmente simétrica. O paciente pode se queixar de tinitus, hipoacusia, fadiga, queda do rendimento laboral, alterações neurovegetativas, estresse e fica sujeito a inúmeras enfermidades orgânicas.

O ruído em excesso tem o poder de lesar considerável extensão das vias auditivas, desde a membrana timpânica até regiões do sistema nervoso central. No órgão de Corti ocorrem as principais alterações responsáveis pela perda auditiva induzida pelo ruído, pois suas células ciliadas externas são particularmente sensíveis a altas e prolongadas pressões sonoras, a chamada "exaustão metabólica”, com depleção enzimática e energética, e redução do oxigênio e nutrientes; com a morte celular, o espaço é preenchido por formações cicatriciais, o que resulta em déficit permanente da capacidade auditiva.

A perda induzida pelo ruído é conseqüência da exposição prolongada a um ambiente ruidoso, existindo dois aspectos fundamentais: as características do ruído e a suscetibilidade individual.
As características do ruído são: intensidade, freqüência, tempo de exposição e natureza do ruído. A intensidade a partir de 84/90 dB de ruído causa uma lesão coclear irreversível e a lesão será mais importante quanto maior for o ruído, o que tem sido razoavelmente comum em alguns ambientes industriais como metalúrgicas, teares, bancos de prova de motores e outros. Na freqüência, qualquer área do espectro sonoro é capaz de desencadear problemas cocleares, tendo como mais traumatizantes os ruídos compostos pelas freqüências altas. Em relação ao tempo de exposição, a lesão é diretamente proporcional ao tempo em que o indivíduo fica exposto ao ruído; com 100 horas de exposição já se pode encontrar patologia coclear irreversível, por este motivo intervalos para descanso acústico em ambientes adequados são fundamentais na tentativa de recuperação enzimática das células sensoriais. A natureza do ruído refere à distribuição da energia sonora durante o tempo, podendo ser contínua, flutuante e intermitente. Ruídos de impacto, como na explosão, são particularmente prejudiciais.

A suscetibilidade individual está relacionada com o sexo, idade e doenças do ouvido. O sexo masculino apresenta preponderância na incidência e no grau de perda auditiva. A idade é importante, pois os mais jovens e os mais idosos apresentam maior suscetibilidade. Doenças do ouvido com disacusia neurossensorial de qualquer etiologia podem significar maior prejuízo ao paciente submetido ao ruído.

O diagnóstico da PAIR deve ser realizado através da obtenção de uma história de exposição em ambiente ruidoso, exame otorrinolaringológico com ênfase na otoscopia e exame audiométrico tonal limiar. As freqüências avaliadas são as tradicionais acrescidas de 3 e $6 \mathrm{kHz}$. O trabalhador avaliado deve realizar, antes do exame, pelo menos 14 horas de repouso acústico, a fim de ser evitada a detecção de uma perda auditiva temporária.

Seligman ${ }^{13}$, 1994, caracteriza a PAIR como perda auditiva sempre neurossensorial, irreversível e quase sempre similar bilateralmente, que raramente leva à perda auditiva profunda pois, geralmente, não ultrapassa os 40 dB nas baixas freqüências e os 75 dB nas altas freqüências, manifestando-se primeira e predominantemente, nas freqüências de 3,4 e $6 \mathrm{kHz}$ e com o agravamento da lesão estendendo-se às freqüências de $8 ; 2 ; 1 ; 0,5$ e 0,25 $\mathrm{kHz}$, as quais levam mais tempo para serem comprometidas. O portador de PAIR pode apresentar intolerância a sons intensos, zumbidos além de comprometimento da inteligibilidade da fala, com prejuízo no processo da comunicação. Não deverá haver progressão da PAIR uma vez cessada a exposição ao ruído intenso. A PAIR geralmente atinge o seu nível máximo para as freqüências de 3, 4 e $6 \mathrm{kHz}$ nos primeiros 10 a 15 anos de exposição sob condições estáveis de ruído.

Relata que a PAIR pode agravar através da exposição 
simultânea do trabalhador a ruídos intensos e outros agentes, tais como produtos químicos e vibrações. Da mesma forma, o trabalhador que ingere ototóxicos ou é portador de algumas doenças pode ter sua suscetibilidade ao ruído aumentada.

$\operatorname{Costa}^{3}, 1994$, refere que os efeitos dependem da intensidade e duração da exposição ao ruído, sendo melhor conhecidos e quantificados o dano causado apenas ao aparelho auditivo, mas existem aspectos extra-auditivos, como alterações físicas e psíquicas causadas pelo ruído.

O ruído elevado poderá atuar como fator predisponente à ocorrência de acidentes do trabalho, segundo Leite e Souto5, 1996 , e ser causa agravante de doenças nos sistemas cardiovascular e endócrino por ação do sistema nervoso autônomo.

O ruído ocupacional é um perigoso agente poluente, segundo Seligman', 1997, sendo o mais comum, entre tantos encontrados na atividade industrial. Os sintomas auditivos geralmente são representados por: perda auditiva, zumbidos, dificuldades na compreensão da fala. Os sintomas extra-auditivos são alterações do sono e transtornos: da comunicação, neurológicos, vestibulares, digestivos, comportamentais, cardiovasculares e hormonais.

A importância da exposição ao ruído no ambiente de trabalho e a ocorrência de perda auditiva induzida pelo ruído principalmente em metalúrgicas é referido por Benevides ${ }^{2}, 1997$.

A necessidade de utilização de protetores auditivos para a redução mecânica do ruído e a de Programas de Conservação Auditiva nas indústrias em que os trabalhados estão expostos a ruído excedendo $85 \mathrm{~dB}$ é relatado por Lusk $^{6}, 1997$.

Nos Programas de Conservação Auditiva, conforme Nepomuceno9, 1997 , são importantes as medições acústicas envolvendo pressão sonora e tempo para determinação dos níveis de exposição a ruído. As informações obtidas com o monitoramento pessoal são fundamentais para a seleção dos protetores auditivos individuais, assim como para o controle e análise das audiometria ocupacionais.

A ocorrência de potencialização devido à interação entre exposição a agentes químicos e ruído é referida por Morata $^{8}, 1998$.

Esta pesquisa objetivou identificar a ocorrência de perda auditiva induzida pelo ruído e os principais sintomas otológicos e a utilização de equipamento de proteção individual, em trabalhadores de metalúrgica do município de Goiânia no período de janeiro a março de 2000.

\section{CASUÍSTICA E MÉTODO}

Após aprovada por comissão de ética médica, a pesquisa foi realizada em indústria metalúrgica do município de Goiânia durante o período de janeiro a março de 2000.
Para a pesquisa foram selecionados 187 trabalhadores com posto de trabalho em ambiente ruidoso, ruído acima de $85 \mathrm{~dB}$, adultos com idade entre 18 e 50 anos, avaliados através de protocolo de triagem composto por roteiro de entrevista e audiometria ocupacional.

O roteiro de entrevista apresentava duas partes: a identificação e a triagem auditiva, visando investigar as principais queixas otológicas do trabalhador. Identificação - obtendo nome, idade, sexo, função, tempo na firma, horário de trabalho e uso de protetores auriculares. Triagem auditiva - questionando sintomas otológicos, doenças sistêmicas e antecedentes; sintomas otológicos como: hipoacusia, dificuldade de compreensão da fala, tinitus, otalgia, sensação de plenitude auricular, tonturas e outros; doenças sistêmicas como Diabetes, Sífilis, Sarampo, Caxumba, Meningite, Tuberculose, Hipertensão Arterial Sistêmica, Doenças da Tireóide, Doenças Renais e outras que podem estar relacionadas com presenças de perda auditiva. Antecedentes de trauma acústico, emprego anterior com ruído, antecedente familiar de surdez, uso de ototóxicos, trabalho com solventes, tabagismo, uso de medicação diária e outros fatores que podem influenciar a audição.

Realizada avaliação otorrinolaringológica com otoscopia em todos os avaliados, sendo normal em todos os submetidos à audiometria ocupacional.

A audiometria tonal foi realizada nas freqüências de $250 \mathrm{~Hz}, 500 \mathrm{~Hz}, 1 \mathrm{kHz}, 2 \mathrm{kHz}, 4 \mathrm{kHz}, 6 \mathrm{kHz}$ e 8 kHz, por uma equipe de duas fonoaudiólogas utilizando audiômetro calibrado da marca Maycos, em todos os trabalhadores selecionados.

As audiometrias foram classificadas, segundo Otacílio e Campos ${ }^{10}$, 1994, como: nível de audição normal até 25 dB, disacusias: leve de $25 \mathrm{~dB}$ até $40 \mathrm{~dB}$, moderada de 45 até 70 $\mathrm{dB}$, severa de $75 \mathrm{~dB}$ até $90 \mathrm{~dB}$, profunda maior que $95 \mathrm{~dB}$.

As audiometrias ocupacionais foram classificadas em três grupos: Audiometrias Normais, Audiometrias sugestivas de Perda Auditiva Induzida pelo Ruído e Audiometrias devido a outras doenças auditivas. Audiometrias sugestivas de Perda Auditiva Induzida pelo Ruído: todas aquelas que apresentam laudo de disacusia neurosensorial leve representando um entalhe em $4 \mathrm{kHz}$ e $6 \mathrm{kHz}$ e disacusia neurosensorial simétrica bilateral variando de nível leve e moderada com alguma freqüência em nível severo. Normais: todas as audiometrias cujos limiares foram no máximo até o nível de $25 \mathrm{~dB}$. Audiometrias devido a outras doenças auditivas: aquelas que apresentavam alterações não relacionadas com Perda Auditiva Induzida pelo Ruído.

\section{RESULTADOS}

Audiometrias ocupacionais sendo: 132 (72\%) audiometrias normais e 41 (21\%) audiometrias sugestivas de PAIR. (Tabela 1; Gráfico 1). 


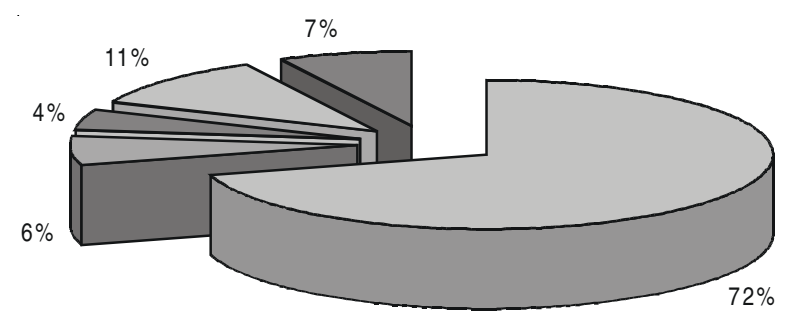

Gráfico 1. Gráfico das Audiometrias Ocupacionais: $72 \%$ normais, $6 \%$ com entalhe em $4 \mathrm{kHz}, 4 \%$ com entalhe em $6 \mathrm{kHz}, 11 \%$ com PAIR e $7 \%$ com outras doenças otológicas.

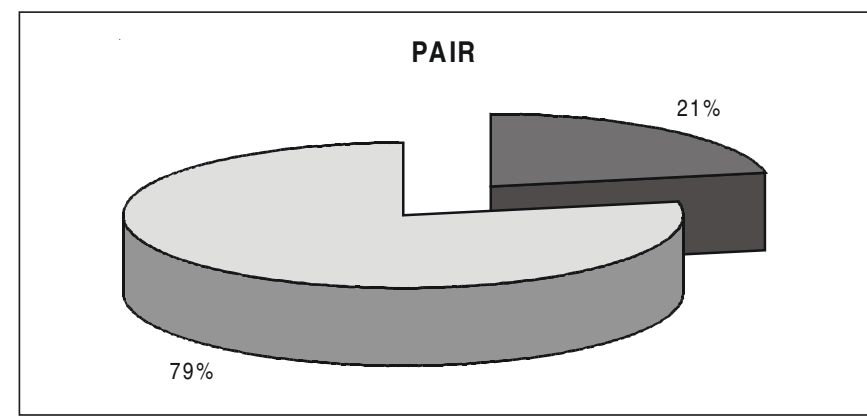

Gráfico 2. Gráfico das Perdas Auditivas sugestivas de PAIR incluindo as perdas auditivas neurossensoriais simétricas e os entalhes em 4 $\mathrm{kHz}$ e $6 \mathrm{kHz}$, totalizando 41 trabalhadores (21\%), sendo $79 \%$ normais.

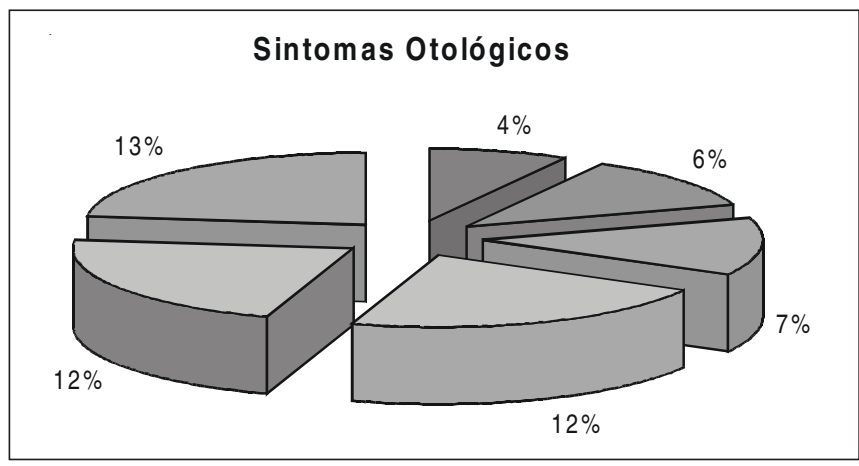

Gráfico 3. Gráfico dos sintomas otológicos mais freqüentes 7\% (13) Hipoacusia, 12\% (22) Dificuldade de compreensão da fala, 12\% (22) Tonturas, 13\% (23) Tinitus, 4\% (8) Sensação de Plenitude Auricular e $6 \%$ (12) Otorreia.

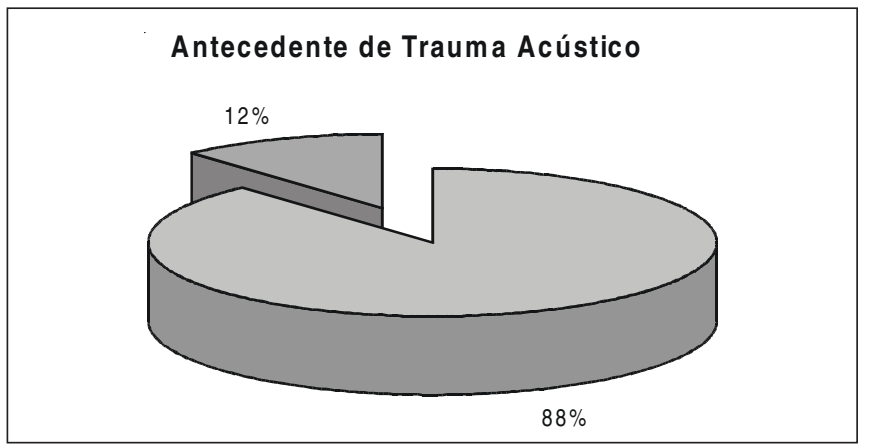

Gráfico 4. Gráfico dos antecedentes de trauma acústico dos trabalhadores $12 \%$
Audiometrias sugestivas de PAIR sendo: 12 (6\%) audiometrias com entalhe em $4 \mathrm{kHz}, 8$ (4\%) audiometrias com entalhe em $6 \mathrm{kHz}$ e 21 (11\%) audiometrias perdas auditivas neurossensoriais sugestivas de perda auditiva induzida pelo ruído PAIR (Tabela 1; Gráfico 1; Gráfico 2). Audiometrias sugestivas de outras doenças auditivas que não PAIR são 14 (7\%) (Tabela 1; Gráfico 1).

Sintomas auditivos com maior freqüência foram: 22 (12\%) dificuldade de compreensão da fala, 13 (7\%) hipoacusia, $23(13 \%)$ tinitus, 8 (4\%) sensação de plenitude auricular, 12 (6\%) otorreia e 22 (12\%) tonturas (Tabela 2; Gráfico 3).

Foi relevante o antecedente de trauma acústico referido por 22 (12\%) dos trabalhadores (Gráfico 4).

Quanto ao uso de Equipamento de Proteção individual tipo abafador auditivo referido por 158 (85\%) dos trabalhadores. (Gráfico 5).

Tabela 1. Tabela das audiometrias Ocupacionais com número total dos resultados e percentagem em relação ao total de trabalhadores.

\begin{tabular}{lcc}
\hline $\begin{array}{l}\text { Audiometrias } \\
\text { Ocupacionais }\end{array}$ & $\begin{array}{c}\text { Número } \\
\text { Total }\end{array}$ & $\begin{array}{c}\text { Percentagem } \\
(\%)\end{array}$ \\
\hline Normal & 132 & 72 \\
PAIR & 21 & 11 \\
Com entalhe em $4 \mathrm{kHz}$ & 12 & 6 \\
Com entalhe em $6 \mathrm{kHz}$ & 8 & 4 \\
Outras doenças Otológicas & 1 & 7 \\
\hline
\end{tabular}

Tabela 2. Tabela dos Sintomas auditivos com o número de trabalhadores com a queixa e sua percentagem em relação ao total de trabalhadores.

\begin{tabular}{lcc}
\hline $\begin{array}{l}\text { Sintomas } \\
\text { Auditivos }\end{array}$ & $\begin{array}{c}\text { Número de } \\
\text { trabalhadores }\end{array}$ & $\begin{array}{c}\text { Percentagem de } \\
\text { trabalhadores (\%) }\end{array}$ \\
\hline Hipoacusia & 13 & 7 \\
Dificuldade de compreensão da fala & 22 & 12 \\
Tinitus & 23 & 13 \\
Tonturas & 22 & 12 \\
Sensação de Plenitude Auricular & 8 & 4 \\
Otorreia & 12 & 6 \\
\hline
\end{tabular}

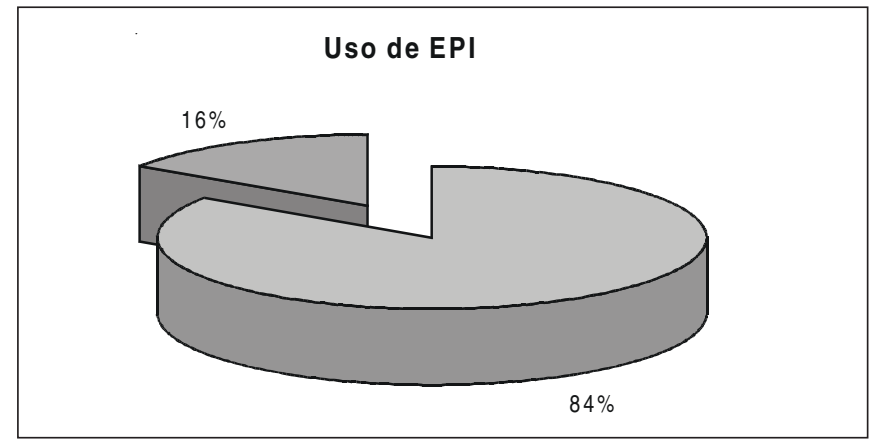

Gráfico 5. Gráfico dos trabalhadores que referem uso de EPI Equipamento de proteção individual abafador auditivo $84 \%$. 


\section{DISCUSSÃO}

A PAIR é um comprometimento auditivo passível de prevenção que pode produzir alterações importantes no trabalhador que interferem na sua qualidade de vida, produzindo desvantagens e incapacidade auditiva. A incapacidade auditiva através da redução da percepção da fala em ambientes ruidosos, televisão, rádio, cinema, teatro, sinais sonoros de alerta, músicas e sons ambientais. Desvantagens sendo conseqüências não-auditivas influenciadas por fatores psicossociais e ambientais como estresse, ansiedade, isolamento e auto-imagem pobre, as quais comprometem as relações do indivíduo na família, no trabalho e na sociedade, prejudicando o desempenho de suas atividades de vida diária.

A perda auditiva relacionada com o trabalho, diferente do trauma acústico, é uma diminuição gradual da acuidade auditiva, decorrente da exposição continuada a níveis elevados de ruído.

A preocupação com a proteção do trabalhador em relação ao ruído é de longa data (desde Mocelin, 1951); é levantada a necessidade de profilaxia individual com uso de proteção auditiva e redução do ruído no ambiente de trabalho.

Os mecanismos que atuam na lesão da cóclea e produzem a PAIR foram estudados por Lim e Dunn', 1979, explicando fisiopatologicamente que a freqüência mais afetada é $4 \mathrm{kHz}$, sendo observado o comprometimento inicial em 4 e $6 \mathrm{kHz}$ nos resultados obtidos nas audiometrias ocupacionais.

A ansiedade expressada pelo aumento das reclamações de sintomas auditivos nos trabalhadores que apresentam diagnóstico sugestivo de PAIR concorda com Russo $^{11}$, 1993, que cita alteração do bem-estar físico e mental dos trabalhadores submetidos a níveis críticos de ruído.

Durante o levantamento audiométrico foi possível relacionar exposição ao ruído com perda auditiva caracterizada por disacusia neurossensorial simétrica como referido por Seligman e Ibañez ${ }^{12}$, 1993, e Seligman ${ }^{13}$, 1994, assim como os sintomas de tinitus e hipoacusia. Outro ponto importante é a necessidade do repouso auditivo de 14 horas antes do exame de audiometria ocupacional para evitar a detecção de uma perda auditiva temporária.

A pesquisa reforça que os efeitos auditivos dependem da intensidade e duração da exposição ao ruído como referido por $\operatorname{Costa}^{3}, 1994$, assim como a ocorrência de alterações físicas e psíquicas causadas pelo ruído.

Não foi possível relacionar o agravamento de doenças nos sistemas cardiovascular e endócrino por ação do ruído no sistema nervoso autônomo como referido por Leite e Souto 5 , 1996, mas foi referido pelos trabalhadores o ruído como fator predisponente à ocorrência de acidentes do trabalho.
O ruído foi referido pelos trabalhadores como um agente poluente na atividade industrial como relatado por Seligman', 1997, sendo também encontrados sintomas auditivos como perda auditiva, zumbidos e dificuldades na compreensão da fala. Foram citados como sintomas extra-auditivos alterações do sono e transtornos da comunicação e neurológicos, vestibulares, como tonturas, e comportamentais, como nervosismo e estresse.

Foi identificada a importância da exposição ao ruído no ambiente de trabalho de metalúrgicas com ocorrência de perda auditiva induzida pelo ruído como referido por Benevides², 1997.

São de grande importância a utilização de protetores auditivos adequados e os Programas de Conservação Auditiva em Metalúrgicas com controle e análise das audiometria ocupacionais como referido por $\mathrm{Lusk}^{6}$, 1997, e Nepomuceno9, 1997.

Não foi possível identificar efeito de potencialização devido à interação entre exposição a agentes químicos e ruído na metalúrgica como foi referido por Morata ${ }^{8}, 1998$, sendo uma excelente proposta de estudo para o futuro.

A exposição dos trabalhadores ao ruído e suas conseqüências são regulamentadas por dois Ministérios: Trabalho e Previdência Social. Na Portaria 3214 do Ministério do Trabalho, a NR-6 diz que o empregador deve fornecer a proteção individual "sempre que as medidas de proteção coletiva forem tecnicamente inviáveis ou não oferecerem completa proteção”. A NR-7 obriga a realização de exames audiométricos tonais por via aérea nas freqüências de 500, 1000, 2000 e 4000 hertz, a cada seis meses, quando os níveis de ruído forem considerados insalubres, o que é regulamentado pelos Anexos I e II da NR-15 (85 dB - 8 horas, 90 dB - 4 horas etc.) como referido por Seligman e Ibañez ${ }^{12}$, 1993, sendo que com a pesquisa comprovamos a necessidade do estudo também da freqüência de $6000 \mathrm{~Hz}$ nas audiometrias ocupacionais pela grande ocorrência de comprometimento auditivo na mesma neste estudo audiométrico de PAIR.

\section{CONCLUSÕES}

Concluímos que os fatores que produzem surdez precoce em trabalhadores de metalúrgica, devido à perda auditiva induzida pelo ruído são o elevado índice de ruído no ambiente da indústria e a não-utilização regular dos protetores auriculares.

A medição dos níveis de ruído nos postos de trabalho é importante para o redimensionamento da carga horária de trabalho em metalúrgica, assim como para a orientação do tipo de protetor auricular que deve ser utilizado.

O risco aumentado de ocorrência de perda auditiva induzida pelo ruído nos trabalhadores de metalúrgica é importante e ocorre principalmente quando não é realizado 
uso regular e correto de protetores auriculares, sendo necessário realizar campanhas de esclarecimento e motivação para o uso dos mesmos.

A importância da execução de audiometrias ocupacionais nos trabalhadores de metalúrgica, para a prevenção e controle da PAIR e a real necessidade de avaliação das freqüências de 250, 500, 1000, 2000, 4000, 6000 e 8000 hertz.

Existe uma relação importante entre as perdas auditivas obtidas nas audiometrias ocupacionais e os sintomas auditivos mais freqüentes como: dificuldade de compreensão da fala, hipoacusia, tinitus, sensação de plenitude auricular, otorreia e tonturas.

\section{REFERÊNCIAS BIBLIOGRÁFICAS}

1. BEHRENS, T.R.; LIEBERGOTT, H.V. Education of the deaf - The otolaryngologist's role. Otolaryngology Clinics of North America, 4(2):423-30, 1971.

2. BENEVIDES, R. - Neurosensorial hearing loss caused by noises: results of a longitudinal study in iron and steelworkers. Ver. Med. Chil., 25:1026-31, 1997.

3. COSTA, V. H. C. - O ruído e suas interferências na saúde do trabalhador. Revista da SOBRAC, 13:912-26, 1994.
4. COMITÊ NACIONAL DE RUÍDO E CONSERVAÇÃO AUDITIVA Recomendações para a avaliação dos prejuízos ocasionados pela Perda Auditiva Induzida pelo Ruído, Carta aos editores, ACTA AWHO, 16:45, 1996.

5. LEITE, J. C. B.; SOUTO, D. F. - Avaliação das perdas auditivas induzidas por exposição a níveis elevados de pressão sonora no ambiente de trabalho, Anteprojeto de Instrução Normativa, Rio de Janeiro, 1996.

6. LUSK, S.L. - Noise exposures. Effects on hearing and prevention of noise induced hearing loss. AAOHN, 45:397-408, 1997.

7. MOCELliN, L. - Profilaxia dos traumatismos sonoros na surdez profissional. Tese para concurso à livre docência da cadeira de clínica otorrinolaringológica da Faculdade de Medicina da Universidade do Paraná, Curitiba, 1951.

8. MORATA, T.C. - Assessing occupational hearing loss: beyond noise exposures. Scand. Audiol. Suppl., 48:111-6, 1998.

9. NUDELMANN, A. A.; COSTA, E.A.; SELIGMAN, J.; IBAÑEZ, R.N. PAIR Perda Auditiva Induzida pelo Ruído. Bagaggem Comunicação, Porto Alegre, 1997.

10. OTACÍLIO, L.F.; CAMPOS,C.A.H. - Tratado de Otorrinolaringologia, Roca, São Paulo, 1994

11. RUSSO, I. C. P. - Ruídos, seus efeitos e medidas preventivas. In: Acústica e psicoacústica aplicada à fonoaudiologia. São Paulo, Lovise, p. 123-38, 1993.

12. SELIGMAN, J.; IBANEZ, R.N. - Considerações a respeito da perda auditiva induzida pelo ruído. ACTA AWHO, 12:75-9, 1993.

13. SELIGMAN, J. - Perda auditiva induzida pelo ruído relacionada ao trabalho. ACTA AWHO, 13:126-7, 1994 